\title{
As Relações entre as Dimensões do Autoconceito e o Comportamento de Compra Masculino
}

\author{
The Relationships Between the Dimensions of Self-Concept and \\ Male Consumption Behavior
}

Carlos Alberto Mello Moyano

Professor Doutor. Universidade de Santa Cruz do Sul (UNISC). Santa Cruz do Sul, RS. Brasil. E-mail: imur@imur.com.uy

Jorge Francisco Bertinetti Lengler

Professor Pós-Doutor. Universidade de Santa Cruz do Sul (UNISC). Santa Cruz do Sul, RS. Brasil. E-mail: jorge@lengler.org

Derli Luís Angnes

Mestre em Administração. Universidade de Santa Cruz do Sul (UNISC). Santa Cruz do Sul, RS. Brasil. E-mail: derli.angnes@gmail.com

\section{Resumo}

O objetivo central deste estudo é analisar a relação de multidependência existente entre o autoconceito do homem e seu comportamento como consumidor. Para alcançar os objetivos propostos por este estudo, foi realizada uma pesquisa de caráter exploratório descritivo. Na fase exploratória, de natureza qualitativa, buscou-se identificar as dimensões do autoconceito do homem $(n=30)$. Na segunda fase, de caráter quantitativo, foram realizadas 403 entrevistas por meio de um instrumento estruturado de coleta de dados. Para se testar as hipóteses de estudo, empregou-se a modelagem de equações estruturais, além do teste de diferenças de médias. Os resultados obtidos apontam que existe uma diferença significativa entre as duas dimensões estudadas do autoconceito: atual e ideal. Diante dos resultados obtidos, pode-se afirmar que o autoconceito exerce influência sobre o comportamento dos homens como consumidores. Os resultados apresentados neste estudo têm implicações diretas sobre a atuação de executivos de empresas de produtos de consumo.

Palavras-chave: Autoconceito. Comportamento do Consumidor. Compra Masculina. Segmentação. Mercado.

\section{Abstract}

The main purpose of this study is to analyze the relationship of multi-dependency between the men's self-concept and their behavior as consumers. To achieve the purpose of this study, we carried out an exploratory-descriptive research. In the exploratory phase (qualitative), we sought to identify the dimensions of man's self-concept $(n=30)$. In the second phase (of quantitative nature) we carried out 403 interviews using a structured data collection instrument. To test the theoretical hypotheses, we used structural equation modeling, in addition to testing mean differences. These results indicate that there is a significant difference between the two dimensions studied self-concept: actual and ideal. Based on our findings it can be argued that the self-concept influences the behavior of men as consumers. The results of this study have direct implications on the performance of business executives from consumer product companies.

Keywords: Self-Concept. Consumer Behavior. Male Consumption. Segmentation. Market. 


\section{INTRODUÇÃO}

A segmentação de indivíduos, enquanto consumidores, em grupos homogêneos capazes de responder a estímulos específicos de marketing, é uma prioridade do marketing na atualidade. A compreensão das motivações de compra dos consumidores é uns dos caminhos para tal tarefa. Nesse contexto, a utilização de diversas técnicas e instrumentos pelos pesquisadores e executivos é uma das formas de efetuar uma adequada segmentação procurando identificar mercados-alvo além de desenvolver o conhecimento sobre os mesmos. A importância do conhecimento dos clientes nas organizações como forma de aumentar o desempenho e satisfação dos clientes tem ocupado a agenda de pesquisa na atualidade (HARDESTY; BEARDEN 2009; PUCCINELLI et al., 2009). Para segmentar os mercados, as empresas podem adotar três abordagens:

a) Marketing de massa, quando há a decisão de produzir e distribuir em massa um produto $e$ tentar atrair todos os tipos de compradores.

b) Marketing de variedade de produtos, no qual a empresa procura oferecer uma variedade de produtos para ampliar a base de clientes.

c) Marketing de mercado-alvo, cuja decisão deve distinguir os diferentes grupos que compõem um mercado para desenvolver produtos correspondentes e compostos de marketing (mix de marketing) para cada mercado-alvo.

Para Etzel, Walker e Station (2001, p. 160), os três passos básicos do marketing de mercado-alvo são a segmentação de mercado, a definição de mercados-alvo e o posicionamento de mercado.

A segmentação de mercado é o ato de dividir um mercado em grupos distintos de compradores com diferentes necessidades ou respostas. Como base para segmentação de mercado, os autores utilizam elementos geográficos, demográficos, psicográficos, comportamentais e multiatributos.

O autoconceito é um tipo de segmentação psicográfica. O conceito de "eu" tem uma longa história na psicologia (PERVIN, 2004), em alguns casos o "eu" foi definido como atitudes e sentimentos da pessoa em relação a si mesma e, em outros casos, como um conjunto de processos psicológicos que governa o comportamento do indivíduo (HALL; GARDNER, 1978). O primeiro, conhecido como "eu como objeto", relacio- na-se claramente com o conceito rogeriano do "eu", ao passo que o segundo, chamado de "eu como processo", relaciona-se com o conceito freudiano do ego.

Dada a importância do autoconceito para compreender os consumidores e sua motivação em ambientes de compras, a pesquisa procurou responder: quais são as relações entre autoconceito e consumo? Para tanto, o primeiro tema a estudar no artigo é justamente a identificação das dimensões que integram o autoconceito masculino, de modo que as dimensões identificadas na pesquisa possam subsidiar e contribuir para o aprimoramento de futuras pesquisas e estudos no âmbito acadêmico e gerencial. O segundo tema deste estudo consiste em verificar a importância dessas variáveis de autoconceito no comportamento de compra nos consumidores brasileiros do sexo masculino. Portanto, acredita-se que o autoconceito e o comportamento do consumidor possuem uma relação de causalidade. Acredita-se também que a análise das relações dos dois construtos pode ajudar a compreender o comportamento de compra. A análise dessas relações é, de fato, a contribuição oferecida por este artigo.

Portanto, o objetivo central do presente estudo é analisar a relação de multidependência existente entre o autoconceito dos homens e seu comportamento enquanto consumidores. Acredita-se que a análise destas relações é capaz de desvelar os antecedentes do comportamento de compra em ambientes de consumo. A análise desta relação é, de fato, a contribuição deste artigo para a compreensão do comportamento de consumo dos homens. Para tanto, dividiu-se o estudo em três partes: na primeira, identifica-se o conjunto de dimensões que integram o "eu atual" e o "eu ideal" dos homens (ROGERS, 1959); posteriormente, avalia-se o comportamento de consumo dos participantes do estudo para, finalmente, avaliar a relação de multidependência existente entre o autoconceito e o comportamento de consumo dos homens entrevistados.

\section{Referencial Teórico e Hipóteses de Pesquisa}

Os conceitos estruturais mais importantes da teoria de Rogers (1959) são o self e o self ideal. O self é uma configuração organizada das percepções e os significados particulares referentes à parte do campo 
fenomênico conhecida como o "eu". O self ideal é o conceito de si mesmo que uma pessoa gostaria de possuir (LANDON, 1974). O "eu" ideal abrange as percepções e os significados potencialmente relevantes para o "eu" e altamente valorizados pelo indivíduo. Essa teoria coloca a ênfase no mundo fenomênico do indivíduo cuidando das diferenças individuais e do caráter global e totalizante da personalidade individual $e$ ainda salienta a maneira em que é percebido $e$ experimentado o mundo.

Tal teoria argumenta que o indivíduo tende à autoconsistência, que é definida como a congruência entre as diversas dimensões do self. Esse processo e esta mudança são os focos da teoria (BISCHOF, 1977), que, principalmente, focaliza no processo e na mudança, indicando que a tendência fundamental de todo indivíduo é o impulso à autoatualização, isto é, a tendência de manter, expandir e atualizar o organismo do experimentador. A percepção no nível subliminal de experiências que têm sido previamente associadas à perda da consideração positiva leva ao estado da incongruência. O processo de eliminar a incongruência e reduzir a ansiedade acontece mediante a utilização dos mecanismos defensivos da personalidade. $\mathrm{O}$ autoconceito é de natureza configuracional e representa uma gestalt conceitual organizada e consistente, um padrão de percepções afins que serve como instrumento de adaptação social.

A análise do autoconceito consiste num eixo de pesquisa por meio do qual o pesquisador identifica a influência de aspectos internos sobre o sistema de ação do indivíduo, que se manifesta desde o comportamento de compra e das atitudes em relação a produtos e serviços, até o comportamento em geral. Entretanto, Reed II (2002) argumenta que a pesquisa do autoconceito caracteriza-se por ser fragmentada e difusa.

Com a finalidade de medir o autoconceito, alguns estudos utilizaram escalas de Likert e outros, entre tantos, adaptaram escalas de diferencial semântico ou a autodescrição ou ainda a escala de autoconceito estatístico (CHAPLIN; JOHN, 2005; CORONA, 2001; DA SILVA; VENDRAMINI, 2005; MIRANDA, 1998).

Alguns estudos afirmam que uma congruência entre o self e a imagem dos produtos pode influenciar as preferências do consumidor (MEHTA, 1999), outros que os valores expressivos do produto permitem uma maior probabilidade de persuasão (FAM; WALLER,
2004) e outros ainda, que o tamanho da amostra deve ser adequado (MEENAI et al., 2007). A partir desses conceitos, pode-se concluir que o êxito dos produtos dependerá da avaliação que faz o consumidor da probabilidade de permitirem que ele percorra a distância entre o self atual e o self ideal, já que as marcas são associadas pelos consumidores com características de personalidade, idêntico às do ser humano e esta faceta permite o desenvolvimento de preferências e compras de marcas ou produtos (HOGG; COX; KEELING, 2000).

A teoria do autoconceito (self e self ideal) tem ocupado a agenda dos pesquisadores de marketing. Vários trabalhos buscaram avaliar a relação entre o autoconceito e aspectos do indivíduo enquanto consumidor (MEENAI et al., 2007; MEHTA, 1999). A teoria do autoconceito tem sua origem com os estudos de Rogers (1959), que serve de alicerce para a compreensão do comportamento do consumidor. Em sua obra, Rogers (1959) propõe a existência de um "eu", ou o autoconceito. Nesse sentido, avaliar o "eu" permite ao pesquisador verificar e avaliar a forma como o indivíduo se compreende perante o mundo. Dessa forma, busca-se entender como esse marco teórico permite uma melhor compreensão do comportamento do consumidor.

Com base nos estudos citados e, a partir da hipótese de que o autoconceito é antecedente para o comportamento de consumo dos indivíduos em situações específicas, propôs-se que a primeira hipótese deste estudo esteja relacionada com as duas dimensões do autoconceito: atual e ideal.

A hipótese $\mathrm{H} 1$ encontra amparo na literatura apresentada anteriormente, que afirma que o indivíduo tende à autoconsistência, isto quer dizer que deve existir uma congruência entre as duas dimensões do self estudadas: a atual e a ideal. Caso a amostra de estudo não apresente resultados diferentes nas duas dimensões, será concluído que os indivíduos atingiram um estado de equilíbrio. Portanto, tem-se a primeira hipótese de estudo:

a) $\mathrm{H} 1$ - Os resultados das duas dimensões avaliadas, self atual e ideal, serão diferentes.

A segmentação de mercados com base no autoconceito fornece um instrumento para a criação de ações de marketing eficientes. O autoconceito tem sido utilizado em diferentes pesquisas com a finalidade de 
avaliar o comportamento de consumo em função deste conceito. No presente estudo, as variáveis de autoconceito, medidas em duas dimensões, self (atual e ideal), foram agrupadas em quatro construtos (F1, F2, F3 e F4), a partir da diferença entre as dimensões, salientando que uma alta diferença entre o autoconceito atual $e$ ideal indica um grau de insatisfação consigo mesmo. Os quatro construtos são os seguintes: o construto F1 vinculado à relação de temas de ordem da vida privada; o construto F2 trata sobre o vínculo com sua mulher; o construto F3 sobre temas de conquista de uma mulher; $e$, finalmente, o construto $\mathrm{F} 4$ é vinculado ao individualismo.

As quatro hipóteses seguintes encontram sustentação nos resultados obtidos no estudo de Mehta (1999), que afirma que uma congruência entre o selfe a imagem dos produtos pode influenciar as preferências do consumidor.

O autoconceito é, na realidade, mais do que um conceito, é um conjunto de conceitos, de representações, de juízos descritivos e valorativos a respeito do próprio sujeito (FIERRO, 1981). O conjunto de conceitos refere-se ao próprio corpo, ao próprio comportamento, à própria situação e as relações sociais. A partir dessa autoconstrução resultante das interações sociais (HARTER, 1996) foram desenvolvidas as hipóteses H2 e H3:

b) H2 - O autoconceito vinculado à ordem na vida privada dos homens influencia positivamente seu comportamento de compra, tendendo o indivíduo a consumir mais.

c) H3 - O autoconceito masculino em relação ao vínculo com sua mulher influencia positivamente seu comportamento de compra, tendendo o indivíduo a consumir mais.

Segundo Sheth, Mittal e Newman (2001), os autoconceitos influenciam profundamente o consumo dos indivíduos e um exemplo disso é a vestimenta, já que elas transmitem símbolos vinculados ao tipo de pessoa que está dentro dela, em termos de status (GOLDSMITH; FLYNN; MOORE, 1999). Portanto, a procura dos símbolos, antes ditos, levará ao homem, entre outros comportamentos, ao consumo. Desta forma:

d) $\mathrm{H} 4$ - O autoconceito masculino vinculado a temas de conquista de uma mulher influencia positivamente seu comportamento de compra, tendendo o indivíduo a consumir mais.
Segundo Aaker (1999), os consumidores escolhem produtos que combinam com seu autoconceito, desta maneira:

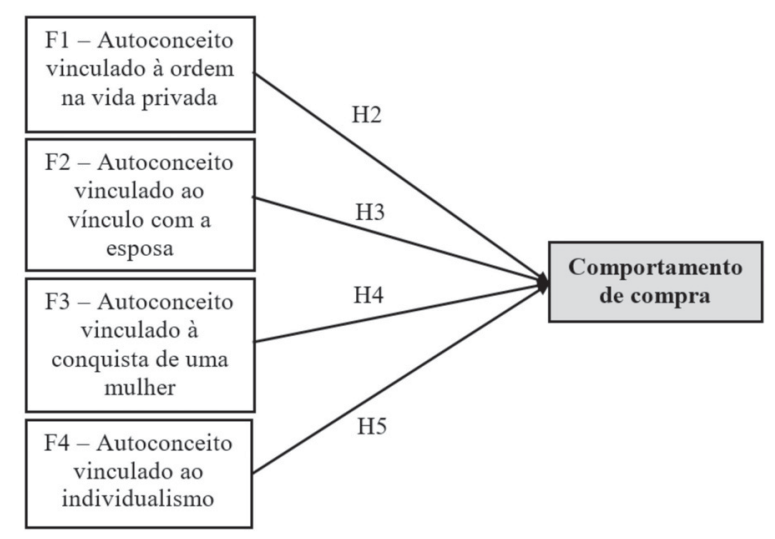

Figura 1: Modelo teórico

Fonte: Elaborada pelos autores deste artigo

\section{Procedimentos Metodológicos}

Para alcançar os objetivos propostos por esse estudo realizou-se uma pesquisa de caráter exploratório descritivo, considerando que, para explicar a base da tomada de decisão individual e o comportamento subsequente, o pesquisador precisa identificar as variáveis mais importantes para a pessoa e aplicar uma técnica sistemática para medi-las. É por isso que a pesquisa de marketing, baseada somente em variáveis escolhidas pelo pesquisador e mensuradas de maneira objetiva, em vez de incluir variáveis identificadas pelo próprio indivíduo e medidas de forma subjetiva, corre o risco de incidir em dois tipos de erros:

a) O uso de variáveis irrelevantes.

b) O uso de mensuração incorreta ou inadequada.

O estudo exploratório, segundo Malhotra (2010), tem como principal objetivo aumentar o conhecimento sobre o tema pesquisado, clarificando conceitos $e$ fornecendo subsídios para as etapas subsequentes da investigação. A pesquisa exploratória é importante para as situações em que o pesquisador não dispõe de informações suficientes para executar o projeto de pesquisa e é caracterizada pela flexibilidade e versatilidade com respeito aos métodos. A pesquisa descritiva caracteriza-se por ser um tipo de pesquisa conclusiva que tem como principal objetivo a descrição de algo, 
normalmente características ou funções de mercado (MALHOTRA, 2010).

$\mathrm{Na}$ fase exploratória, de natureza qualitativa, buscou-se identificar as dimensões do "eu", além de permitir ao pesquisador um maior grau de conhecimento a respeito do tema em foco. Nesta primeira etapa, optou-se por empregar na identificação das dimensões do autoconceito uma adaptação do Incidente Crítico de Flanagan (1954), no qual os respondentes, os homens, são convidados a indicar quais são os elementos que definem um homem e quais seriam os elementos que não os definem. Nessa etapa qualitativa, o questionário foi aplicado a 30 respondentes, sendo: 15 homens e 15 mulheres, $50 \%$ deles professores e $50 \%$ estudantes, todos eles da área das Ciências Aplicadas, escolhidos por conveniência do pesquisador. Foram identificadas 72 dimensões. Após a análise feita por três juízes, decidiu-se selecionar 32 dimensões que foram utilizadas na etapa seguinte. A análise efetuada pelos juízes foi realizada de acordo com as etapas descritas por Hayes (2008). Os resultados dessa etapa permitiram responder a três perguntas formuladas por Vieira $e$ Zouain (2004), que possibilitaram complementar e aprofundar o conhecimento do objeto de estudo - o homem. Em primeiro lugar, oferecendo elementos para a formulação das hipóteses da etapa seguinte; em segundo lugar, aumentando o conhecimento das dimensões do objeto de estudo e; em terceiro lugar, ajudando a desenvolver o questionário a ser aplicado na etapa seguinte.

Na segunda etapa foi construído um questionário a partir das 32 dimensões, identificadas na etapa anterior e aplicado em entrevistas pessoais para uma amostra não probabilística envolvendo 403 homens que residem na Região Sul do Brasil, sendo a amostragem por conveniência do pesquisador. As entrevistas foram realizadas nos domicílios ou nos lugares de trabalho dos entrevistados. Na elaboração do instrumento de coleta de campo - um questionário estruturado - para levantar os dados necessários para a consecução da etapa descritiva, cuja natureza é quantitativa, foram utilizadas escalas de diferencial semântico de sete pontos, onde os extremos das escalas representaram os pólos opostos da dimensão. Em cada dimensão, avaliou-se o self atual e o self ideal.

As variáveis de comportamento de compra foram medidas através de escalas de intensidade, onde os en- trevistadores manifestaram a intensidade de consumo de produtos de higiene (xampu e perfume) em uma escala que variava de não consome (1) até consome muito (7). O questionário foi submetido a um pré-teste, não apresentando dificuldades em sua aplicação ou interpretação das questões por parte dos respondentes.

As variáveis de autoconceito medidas em duas dimensões foram agrupadas em quatro construtos, a partir da diferença entre as dimensões, salientando que uma alta diferença entre o autoconceito atual $e$ ideal indica um grau de insatisfação no consumidor (Figura 1):

a) F1 - com relação a temas vinculados à ordem na vida privada.

b) F2 - temas vinculados ao vínculo com a esposa.

c) F3 - com relação a temas de conquista de uma mulher.

d) F4 - vinculado ao individualismo.

Os quatro construtos reuniram variáveis propostas pela teoria de Rogers (1959) e encontram sustentação nos resultados obtidos no estudo de Mehta (1999). Esses construtos foram obtidos a partir da análise fatorial exploratória (EFA) e itens com carga fatorial inferior a 0,60 foram excluídos.

\section{AnÁlise e INTERPRETAÇÃo dos Resultados}

Nesta seção serão apresentados os resultados das duas fases realizadas: qualitativa e quantitativa. $\mathrm{Na}$ primeira fase, será apresentada a identificação das dimensões do autoconceito e, na segunda, a medição dele.

Na primeira fase foram realizadas entrevistas com informantes qualificados $(\mathrm{n}=30)$, com a finalidade de identificar as dimensões do autoconceito do homem. As dimensões identificadas são $72 e$ as selecionadas, 32. O critério para selecionar as dimensões foi o de incorporar a maior quantidade possível, evitando as repetições. A seguir, desenvolveu-se um questionário estruturado, aplicado na seguinte fase, utilizando escalas de diferencial semântico de sete pontos onde os extremos das escalas representaram os polos opostos da dimensão. Em cada dimensão avaliou-se o self atual e o self ideal. 
Na segunda fase, de caráter quantitativo, foram realizadas 403 entrevistas por meio de um questionário estruturado. A amostra tipo não probabilística foi selecionada conforme a conveniência do pesquisador, mas considerando a formação universitária e a idade, foi composta de 433 entrevistados.

Dos questionários gerados, 30 foram rejeitados, principalmente por não possuírem dados de identificação (11) e por não estarem completadas as perguntas relacionadas ao consumo (19). Após as exclusões foram validados 403 questionários.

As entrevistas foram realizadas em locais de estudo ou de trabalho. A Tabela 1 apresenta a composição da amostra com as características da amostra pesquisada, no que se refere à idade, escolaridade $e$ número de filhos.

Tabela 1: Características da amostra

\begin{tabular}{|c|c|c|}
\hline Características & Percentual (\%) & NúMERo (N) \\
\hline \multicolumn{3}{|c|}{ Escolaridade } \\
\hline $\begin{array}{c}\text { Com formação } \\
\text { universitária }\end{array}$ & 48 & 193 \\
\hline $\begin{array}{c}\text { Sem formação } \\
\text { universitária }\end{array}$ & 52 & 210 \\
\hline \multicolumn{3}{|c|}{ Idade } \\
\hline 18 a 45 anos & 50 & 201 \\
\hline 46 a 70 anos & 50 & 202 \\
\hline \multicolumn{3}{|c|}{ Número de Filhos } \\
\hline Sem filhos & 5 & 20 \\
\hline 1 filho & 27 & 109 \\
\hline 2 filhos & 44 & 177 \\
\hline 3 ou mais filhos & 24 & 97 \\
\hline TOTAL & 100 & 403 \\
\hline
\end{tabular}

Fonte: Dados da pesquisa

\subsection{Modelo de Medida, Validade e Confiabilidade dos Construtos}

Conforme os procedimentos de análise fatorial confirmatória foram avaliados a validade discriminante, convergente e confiabilidade da escala, de acordo com o paradigma defendido por Gerbing e Anderson (1988). A validade de conteúdo foi estabelecida por meio da revisão de literatura e pela consulta a pesqui- sadores da área de comportamento do consumidor. A partir do emprego da teoria e do posicionamento dos pesquisadores em relação ao instrumento utilizado pode-se concluir que as variáveis empregadas no estudo apresentam validade de conteúdo.

Variáveis com correlações inferiores a 0,4 (PRITCHARD; HAVITZ; HOWARD, 1999) foram eliminadas das análises posteriores. Adicionalmente, itens cuja eliminação aumentasse significativamente o coeficiente de alfa de Cronbach também foram eliminados. Com essas ações e após os procedimentos de depuração, todas as variáveis utilizadas no modelo de medida foram consideradas unidimensionais $e$ demonstraram aceitável confiabilidade, com todos os coeficientes alfa de Cronbach superiores a 0,6 (CRONBACH, 1951; HAIR et al., 1998; HU; BENTLER, 1999), demonstrando confiabilidade e consistência nas respectivas medições. Desta forma, os cinco construtos foram considerados suficientes e adequados em termos de como o modelo de medida é especificado. A validade convergente dos construtos é aferida pela carga padronizada das variáveis do modelo de medida $(t>1,96, p<0,05)$ (GERBING; ANDERSON, 1988).

A Tabela 2 indica os coeficientes da variância extraída, alfa de Cronbach e confiabilidade composta dos cinco construtos obtidos no modelo de medida. Os valores de variância extraída também atingiram um mínimo de 0,46 chegando aos 0,96 .

Tabela 2: Análise confirmatória e confiabilidade dos construtos

\begin{tabular}{|c|c|c|}
\hline Construtos E Itens & Carga Fatorial & VALOR $T$ \\
\hline \multicolumn{3}{|c|}{$\begin{array}{l}\text { F1 Autoconceito vinculado à ordem na vida } \\
\text { privada }(\alpha=.76 ; \mathrm{AVE}=.46 ; \rho=.71)\end{array}$} \\
\hline P14 responsável & 0,69 & $---a$ \\
\hline P16 organizado & 0,67 & 2,413 \\
\hline $\begin{array}{l}\text { P31 satisfeito com } \\
\text { minha vida }\end{array}$ & 0,63 & 1,981 \\
\hline P29 centrado & 0,61 & 2,580 \\
\hline P15 realizado & 0,60 & 2,783 \\
\hline \multicolumn{3}{|c|}{$\begin{array}{l}\text { F2 Autoconceito vinculado ao vínculo com a } \\
\text { esposa }(\alpha=.85, \mathrm{AVE}=.66 ; \rho=.82)\end{array}$} \\
\hline P2 dedicado à família & 0,92 & 13,341 \\
\hline P3 compreensivo & 0,73 & 3,427 \\
\hline P5 totalmente feliz & 0,83 & $---a$ \\
\hline
\end{tabular}




\begin{tabular}{|c|c|c|}
\hline P1 romântico & 0,75 & 4,534 \\
\hline \multicolumn{3}{|c|}{$\begin{array}{l}\text { F3 Autoconceito vinculado à conquista de uma } \\
\text { mulher }(\alpha=.67, \mathrm{AVE}=.72 ; \rho=.83)\end{array}$} \\
\hline P23 simpático & 0,71 & 3,220 \\
\hline P26 viril & 0,96 & $---a$ \\
\hline \multicolumn{3}{|c|}{$\begin{array}{l}\text { F4 Autoconceito vinculado ao individualismo } \\
\qquad(\alpha=.68, \mathrm{AVE}=.60 ; \rho=.75)\end{array}$} \\
\hline P6 individualista & 0,72 & 4,237 \\
\hline P24 gosto de cerveja & 0,83 & $---a$ \\
\hline \multicolumn{3}{|c|}{ Comportamento de compra $(\alpha=.88 ; \mathrm{AVE}=.89 ; \rho=.94)$} \\
\hline B233 perfume & 0,93 & 4,986 \\
\hline C233 xampu & 0,94 & $---a$ \\
\hline
\end{tabular}

Notas: $\alpha=$ Alfa de Cronbach (CRONBACH, 1951). $\rho=$ Confiabilidade composta (BAGOZZI, 1980). AVE = Variância média extraída. a = Os valores do teste $t$ para estes parâmetros não são válidos, uma vez que foram previamente fixados.

Fonte: Elaborada pelos autores deste artigo

Pode-se afirmar que os construtos presentes no modelo apresentam validade discriminante, uma vez que a diagonal da matriz (Tabela 3) é maior do que os valores das respectivas colunas e linhas da matriz.

Tabela 3: Correlações entre as variáveis latentes

\begin{tabular}{|c|c|c|c|c|c|}
\hline Construto & $\mathbf{1}$ & $\mathbf{2}$ & $\mathbf{3}$ & $\mathbf{4}$ & $\mathbf{5}$ \\
\hline $\begin{array}{c}\text { 1- Comportamento } \\
\text { de compra }\end{array}$ & 0,94 & & & & \\
\hline $2-\mathrm{F} 1$ & 0,17 & 0,68 & & & \\
\hline $3-\mathrm{F} 2$ & 0,16 & 0,27 & 0,81 & & \\
\hline $4-\mathrm{F} 3$ & 0,14 & 0,28 & 0,25 & 0,85 & \\
\hline $5-\mathrm{F} 4$ & $-0,17$ & $-0,13$ & $-0,07$ & $-0,13$ & 0,77 \\
\hline
\end{tabular}

Nota: A diagonal da matriz é a raiz quadrada dos AVEs

Fonte: Elaborada pelos autores deste artigo

A validade discriminante foi verificada pelas correlações entre os construtos. Essas foram significativamente diferentes de 1 e a variância entre quaisquer dois construtos foi inferior à variância média explicada nos itens do construto (FORNELL; LARCKER, 1981).

O qui-quadrado obtido para o modelo de medida foi de 325,6 ( $p<0,01$ ) com 80 graus de liberdade. Levando em consideração que o qui-quadrado não é uma medida adequada para amostras grandes $(n=$ 403), e que pode levar o pesquisador a conclusões inadequadas, foram empregadas outras medidas de ajustamento (JÖRESKOG; SÖRBOM, 1993) (CFI =
0,971; Tucker-Lewis fit index, TLI $=0,907$; Incremental fit index, IFI $=0,974)$, além do RMSEA $(0,068)$. Os resultados para a análise fatorial confirmatória do modelo de medida indicam que os itens empregados para medir os construtos são válidos e confiáveis.

\subsection{Teste da Hipótese Relacionada à Identificação do Autoconceito Atual e Ideal}

O autoconceito foi medido em duas dimensões: atual e ideal. Na Tabela 4 são expostos os resultados das médias obtidas e os resultados do teste aplicado.

Tabela 4: Resumo de médias das variáveis de autoconceito atual $e$ ideal $(n=403)$

\begin{tabular}{|c|c|c|c|}
\hline Dimensão do Autoconceito & $\begin{array}{l}\text { Atual } \\
\text { “SOU" }\end{array}$ & $\begin{array}{l}\text { Ideal } \\
\text { “desejo } \\
\text { ser” }\end{array}$ & $\begin{array}{l}\text { Teste } \\
t(\%)\end{array}$ \\
\hline Valente & 5,3 & 6,0 & 1 \\
\hline Romântico & 5,1 & 5,7 & 1 \\
\hline Dedicado à família & 5,7 & 6,1 & 1 \\
\hline Compreensivo & 5,3 & 5,9 & 1 \\
\hline Gosto de cozinhar & 4,5 & 5,5 & 1 \\
\hline Sou feliz & 5,9 & 6,4 & 1 \\
\hline Individualista & 4,4 & 3,9 & 1 \\
\hline Criativo & 5,3 & 6,4 & 1 \\
\hline Ativo & 5,2 & 6,1 & 1 \\
\hline Ambicioso & 5,2 & 5,4 & 1 \\
\hline Competitivo & 5,1 & 5,8 & 1 \\
\hline Independente & 5,0 & 6,1 & 1 \\
\hline Batalhador & 5,8 & 6,4 & 1 \\
\hline Exigente & 5,6 & 5,1 & 1 \\
\hline Responsável & 5,6 & 6,4 & 1 \\
\hline Realizado & 5,3 & 6,4 & 1 \\
\hline Organizado & 5,0 & 6,3 & 1 \\
\hline Elegante & 4,5 & 5,8 & 1 \\
\hline Fiel & 5,8 & 6,3 & 1 \\
\hline Tímido & 4,4 & 3,5 & 1 \\
\hline Alegre & 5,7 & 6,3 & 1 \\
\hline Lindo & 4,5 & 5,8 & 1 \\
\hline Gosto de futebol & 5,5 & 5,7 & $\mathbf{1}$ \\
\hline
\end{tabular}




\begin{tabular}{|c|c|c|c|}
\hline Simpático & 5,5 & 6,1 & $\mathbf{1}$ \\
\hline Gosto de cerveja & 5,4 & 5,3 & $\mathbf{1}$ \\
\hline Determinado & 5,7 & 6,4 & $\mathbf{1}$ \\
\hline Viril & 5,0 & 5,3 & $\mathbf{1}$ \\
\hline Vaidoso & 4,7 & 5,1 & $\mathbf{1}$ \\
\hline Inteligente & 5,4 & 6,5 & $\mathbf{1}$ \\
\hline Centrado & 5,1 & 6,1 & $\mathbf{1}$ \\
\hline Ciumento & 4,8 & 4,3 & $\mathbf{1}$ \\
\hline Satisfeito com minha vida & 5,5 & 6,6 & $\mathbf{1}$ \\
\hline
\end{tabular}

Fonte: Dados da pesquisa

Como pode ser observado na Tabela 4, existem diferenças significativas em todas as dimensões estudadas. A partir desses dados, foi possível concluir que o "eu" atual e o ideal são diferentes, indicando que os homens desejam ser diferentes do que que atualmente percebem de si mesmos. O conjunto de resultados apresentados na Tabela 4 permite aceitar a hipótese 1 , na qual se propunha que o self atual e o self ideal dos entrevistados seriam diferentes.

\subsection{Teste das Hipóteses Relacionadas à Influência do Autoconceito sobre o Consumo dos Homens}

Em função da complexidade do modelo estrutural testado e da necessidade de se testar, simultaneamente, as relações estabelecidas entre os construtos, utilizou-se a modelagem de equações estruturais aplicando-se o método de maxiverissimilhança por meio do software Amos 17. O qui-quadrado para o modelo de relações apresentado na Figura 1 foi significativo (qui-quadrado $=325,627, g l=80, p<0,01$ ). Após a análise inicial do ajustamento do modelo examinou-se o ajustamento global a partir dos parâmetros propostos por Bollen (1989) e Byrne (2001). As medidas de ajustamento analisadas foram: $\mathrm{CFI}=0,971$, índice de ajustameto Tucker-Lewis (TLI = 0,907), IFI =0,974, e RMSEA = 0,068 . Uma vez que todas as medidas de ajustamento empregadas se mostraram dentro dos limites estabelecidos pela literatura, o modelo estrutural foi considerado aceitável (BYRNE, 2001; VANDENBERG; LANCE, 2000). As relações teóricas propostas no modelo serão analisadas a seguir.
Os resultados da estimação do modelo indicam que os parâmetros das hipóteses 2 e 3 não foram significativos. Entretanto, os resultados indicam que a hipótese 4, relativa ao autoconceito vinculado a temas de conquista de uma mulher e a relação com o comportamento de compra deve ser aceita, uma vez que o parâmetro obtido foi significativo e positivo $(0,279, t=2,071, p<0,01)$. Tal resultado permite concluir que, quanto maior o autoconceito do homem em relação aos temas vinculados à consquista de uma mulher, mais ele tenderá a consumir. O resultado obtido para a hipótese 5 indica uma relação negativa entre o autoconceito masculino vinculado ao individualismo $e$ o comportamento de compra $(-0,604, t=-2,049, p$ $<0,01)$, resultado oposto àquele esperado. Portanto, a hipótese 5 foi rejeitada. O resultado deste parâmetro permite sugerir que quanto mais individualista o homem se sente, menos ele tenderá a consumir.

Em síntese, os resultados indicam que, das cinco hipóteses testadas, duas foram aceitas ( $\mathrm{H} 1$ e H4), duas obtiveram parâmetros não significativos (H2 e H3) e uma foi rejeitada (H5). A Figura 2 apresenta as relações estruturais e os respectivos resultados obtidos neste estudo.

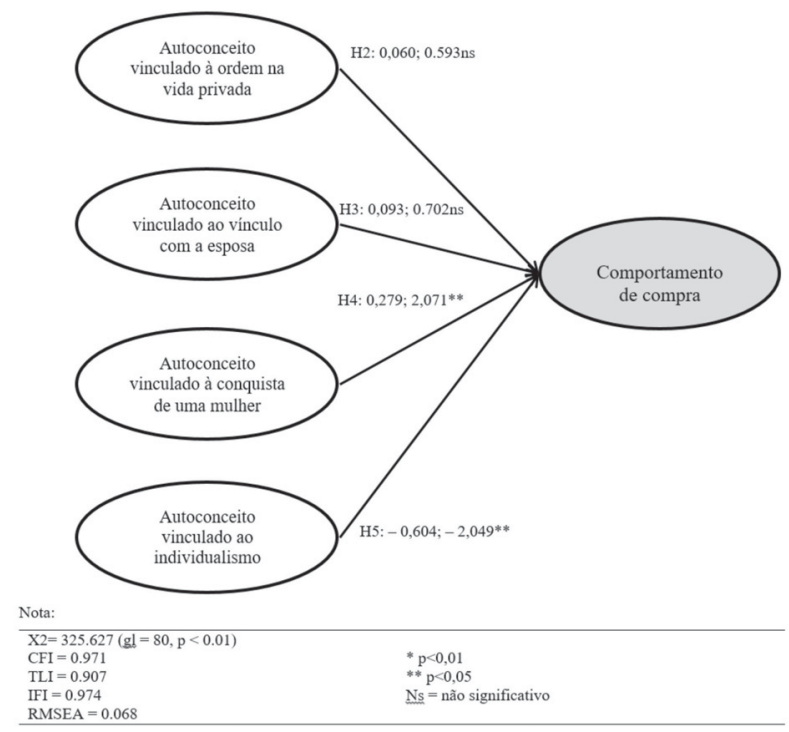

Figura 2: Modelo Estrutural

Fonte: Elaborada pelos autores deste artigo

\section{Considerações Finais}

O propósito fundamental deste artigo foi avaliar o autoconceito dos homens e sua relação com o con- 
sumo. Esse propósito foi definido para que o presente estudo auxilie os gestores da área de marketing a compreender a dinâmica do mercado masculino e a necessidade de adaptação das estratégias de marketing.

Embora se trate de um estudo com homens da Região Sul do Brasil, o estudo pode auxiliar gestores de empresas no esforço de compreensão de seu público-alvo.

Das cinco hipóteses lançadas, duas foram confirmadas e outras três não foram confirmadas. Uma das hipóteses foi rejeitada (H5). A primeira em ser aceita, $\mathrm{H} 1$, demonstra que existem diferenças entre as duas dimensões do self pesquisadas: atual e ideal.

O desenvolvimento de estratégias mercadológicas deve considerar o que os homens são e desejam ser, já que o êxito dos produtos para esse público-alvo está relacionado, provavelmente, à percepção do consumidor de que ele poderá ajudar a percorrer o caminho entre o que ele acredita que é e entre o que ele deseja ser. Nesse caminho do conhecimento do consumidor é preciso salientar os aspectos simbólicos dos produtos e o mundo ideal desse grupo de consumidores e como aqueles permitem ao consumidor chegar ao equilíbrio que ele procura, ainda que seja um instante na vida dele, já que, como dito anteriormente, os selfs se encontram em permanente movimento.

Em relação à influência do autoconceito no consumo, comprovou-se essa relação nas variáveis vinculadas a temas da conquista da mulher, hipótese 4: simpático e viril. Considerando que essas variáveis influenciam significativamente o consumo de perfume e xampu, poder-se-ia salientar que a comunicação desses produtos deveria mostrar aos homens, como sua utilização influencia positivamente em sua relação com as mulheres. Essas comunicações deveriam salientar a virilidade e a simpatia dos homens.

Ao não confirmar as hipóteses 2 e 3 , seria possível dizer que a utilização de certos aspectos do autoconceito masculino na estratégia de marketing e de comunicação do perfume e do xampu não influencia em nada o consumo desse tipo de produto. Esses aspectos são os seguintes: ser responsável, organizado, satisfeito com minha vida, centrado, realizado, dedicado à família, compreensivo, totalmente feliz e romântico.

A rejeição da hipótese 5 e a relação negativa com o consumo revelada pelo resultado do parâmetro testado, resultado diferente ao esperado, devem ser considerados dentro da dinâmica dos selfs atual e ideal. Nas duas dimensões os entrevistados desejavam possuir menos, querendo dizer que eram menos individualistas e gostavam menos de cerveja. Mas por que essa avaliação influencia negativamente o consumo dos produtos estudados? Acredita-se que novas pesquisas poderão aprofundar o entendimento de tal relação.

Apesar de empregar uma amostra de homens de somente uma região do país, o presente estudo colabora para a compreensão da dinâmica do autoconceito e suas relações de consumo apontando a necessidade de adaptação do composto de marketing, a partir da perspectiva do consumidor para o qual o produto é direcionado.

As pesquisas que podem suceder a este estudo assumem múltiplas formas. Uma linha de pesquisa refere-se ao estudo da relação negativa da dimensão individualista do self com o consumo. Outra linha poderia considerar outros contextos culturais com a finalidade de desenvolver uma análise comparativa dos resultados. Ainda, outras linhas de produtos, além daquelas abordadas neste estudo, poderiam ser analisadas. Novas pesquisas sobre o vínculo existente entre o self dos consumidores e a avaliação de marcas representam oportunidades de aprofundamento dos resultados ora apresentados.

\section{REFERÊNCIAS}

AAKER, J. The malleable self: the role of self - expression in persuasion. Journal of Marketing Research, [S.I.], 36, p. 45-57, 1999.

BAGOZZI, R. P. Causal models in Marketing. New York: John Wiley, Inc., 1980.

BISCHOF, S. L. Interpretación de las teorías de la personalidad. México: Trillas, 1977.

\section{BOLLEN, K. A. Structural equations with latent} variables. New York: Wiley, 1989.

\section{BYRNE, B. M. Structural equation modeling with}

Amos: basic concepts, applications, and programming. New Jersey: Lawrence Erlbaum Associates, Inc., 2001.

CHAPLIN, L. N.; JOHN, D. R. The development of selfbrand connections in children and adolescents. Journal of Consumer Research, [S.I.], v. 32, n. 1, p. 119-129, 2005. 
CORONA, L. C. G. Escala reduzida do autoconceito ERA. Manual da Psicologia Aplicada, Rio de Janeiro: CEPA, 2001

CRONBACH, L. J. Coefficient Alpha and the internal structure of tests. Psychometrika, [S.l.], v. 16, n. 3, p. 297-324, 1951.

DA SILVA, M. C. R.; VENDRAMINI, C. M. M. Autoconceito e desempenho de universitários na disciplina de estatística. Psicologia Escolar e

Educacional, [S.l.], v. 9, n. 2, p. 261-268, 2005.

ETZEL, M.; WALKER, B. J.; STATION, W. J. Marketing. São Paulo: Makron Books, 2001.

FAM, K. S.; WALLER, D. S. Ad likeability and brand recall in Asia: a cross-cultural study. Journal of Brand Management, [S.I.], v. 12, n. 2, p. 93-105, 2004.

FIERRO, A. Lecturas de psicologia de la personalidad. Madrid: Alianza Editorial, 1981.

FLANAGAN, J. C. The critical incident technique. Psychological Bulletin, [S.l.], v. 5, n. 4, p. 327-358, 1954.

FORNELL, C.; LARCKER, D. F. Evaluating structural equation models with unobservable variables and measurement error. Journal of Marketing Research, [S.l.], v. 18, n. 1, p. 39-50, 1981.

GERBING, D. W.; ANDERSON, J. C. An updated paradigm for scale development incorporating unidimensionality and its assessment. Journal of

Marketing Research, [S.l.], v. 25, n. 2, p. 186-192, 1988.

GOLDSMITH, R. E.; FLYNN, L. R.; MOORE, M. A. The self-concept of fashion leaders. Clothing and Textiles

Research Journal, [S.l.], v. 14, n. 4, p. 242-8, 1996.

HAIR, J. et al. Multivariate data analysis. New Jersey: Prentice-Hall International, 1998.

HALL, C. S.; GARDNER, L. Theories of personality. 3. ed. New York: Wiley, 1978.

HARDESTY, D. M.; BEARDEN, W. O. Consumer behavior and retailing. Journal of Retailing, [S.I.], v. 85, n. 3, p. 239-244, 2009.
HARTER, S. Historical roots of contemporary issues involving self-concept. In: Handbook of self-concept: developmental, social, and clinical considerations. New York: John Wiley \& Sons, 1996.

HAYES, B. E. Measuring customer satisfaction and loyalty: survey design, use, and statistical analysis methods. 3. ed. Milwaukee: American Society for Quality, 2008.

HOGG, M. K.; COX, A. J.; KEELING, K. The impact of self-monitoring on image congruence and product/brand evaluation. European Journal of Marketing, [S.I.], v. 34, n. 5, p. 641-666, 2000.

HU, L. T.; BENTLER, P. M. Cutoff criteria for fit indexes in covariance structure analysis: conventional criteria versus new alternatives. Structural Equation Modeling: a Multidisciplinary Journal, [S.l.], v. 6, p. 1-55, 1999.

JÖRESKOG, K. G.; SÖRBOM, D. LISREL 8: Structural equation modeling with the SIMPLIS command language. Chicago: Scientific Software International, 1993.

LANDON, L. Self-concept, ideal self-concept and consumer purchase intentions. Journal of Consumer Research, [S.l.], v. 1, n. 2, p. 44-51, 1974.

MALHOTRA, N. Marketing research: an applied orientation. Boston: Pearson, 2010.

MEENAI, Y. A. et al. Value-expressive advertising on women with regard totheir actual self-concept, intention and brand recall. Journal of Management and Social Sciences, [S.l.], v. 3, n. 2, 2007.

MEHTA, A. Using self-concept to assess advertising effectiveness. Journal of Advertising Research, [S.I.], v. 39, n. 1, p. 81-89, 1999.

MIRANDA, A. P. C. Comportamento de consumo de vestuário de moda feminino: uma análise exploratória. Curitiba: Universidade Federal do Paraná, 1998.

PERVIN, L. Personality: theory and research. New Jersey: John Wiley, Inc., 2004.

PRITCHARD, M. P.; HAVITZ, M. E.; HOWARD, D. R. Analyzing the commitment-loyalty link in service contexts. Journal of Academy of Marketing Science, [S.1.], v. 27, n. 3, p. 333-348, 1999,. 
PUCCINELLI, N. et al. Customer experience management in retailing: understanding the buying process. Journal of Retailing, [S.l.], v. 85, n. 1, p. 15-30, 2009.

REED II, A. Social Identity as a useful perspective for self-concept-based consumer research. Psychology \& Marketing, v. 19, n. 3, p. 235, 2002.

ROGERS, C. A theory of therapy, personality, and interpersonal relationships as developer in the clientcentered framework. In S. Koch (Ed.), Psychology: a study of a science. New York: McGraw-Hill, 1959.

SHETH, J. N.; MITTAL, B.; NEWMAN, B. I.

Comportamento do cliente: indo além do comportamento do consumidor. São Paulo: Atlas, 2001.

VANDENBERG, R. J.; LANCE, C. E. A Review and synthesis of the measurement invariance literature: suggestions, practices, and recommendations for organizational research. Organizational Research Methods, [S.l.], v. 3, n. 1, p. 4-70, 2000.

VIEIRA, M. M. F.; ZOUAIN, D. M. Pesquisa qualitativa em administração. Rio de Janeiro: Editora FGV, 2004. 